\title{
Metodologia para design de interfaces digitais para educação
}

\author{
Methodology for design of digital interfaces for education
}

Paula Caroline S. J. Passos, Patricia Alejandra Behar

design, educação, interação, interface

\begin{abstract}
Esta pesquisa apresenta a estruturação de metodologia própria para o design de interfaces de materiais educacionais digitais. A metodologia Interad é proposta com base em pesquisas da área da Educação combinadas a estudos relativos ao design de interação, a arquitetura da informação e ao planejamento visual. Objetiva-se, dessa forma, atender tanto a aspectos educacionais quanto à necessidades técnicas do projeto promovendo a qualidade do produto final. Além da pesquisa bibliográfica dos temas citados, foram ainda levantados dados para a pesquisa por meio de observação participante realizada no Núcleo de Tecnologia Digital Aplicada à Educação, da Universidade Federal do Rio Grande do Sul (UFRGS) em situações práticas de projeto. A metodologia foi validada em curso ofertado pela Secretaria de Educação a Distância (SEAD/UFRGS) onde professores da universidade aplicaram-na em seus projetos de recursos digitais, conforme descreve o presente artigo.
\end{abstract}

design, education, interaction, interface

This research presents the methodology structuring proper for design of digital educational materials interfaces. The Interad methodology is proposed based on researches in Education combined with studies on interaction design, information architecture and visual planning. The goal is to meet both the educational aspects regarding the technical needs of the project promoting the quality of the final product. Besides the bibliographical research of the cited themes, were also collected data for research through participant observation conducted in the Center for Digital Technology Applied to Education, Universidade Federal do Rio Grande do Sul (UFRGS), in practical situations of project. The methodology was validated in a course offered by the Department of Distance Education (SEAD / UFRGS) where university professors have implemented it in their projects of digital resources, as described in this article.

\section{Introdução}

A metodologia Interad (Interfaces Interativas Digitais Aplicadas à Educação) apresenta um processo de desenvolvimento específico para interfaces de materiais educacionais digitais (MEDs). Esta pesquisa considera como MED todo recurso digital elaborado com objetivos relacionados à aprendizagem (Behar, 2009). Entende-se a aprendizagem como o processo no qual o indivíduo constrói o próprio conhecimento através da interação com o meio (Piaget, 1974). A interface é definida aqui como a superfície que possibilita o contato entre usuário e conteúdo, ou entre usuário e usuário (no caso de estarem conectados em rede) e permite o acesso às funções do sistema.

Sendo que o desenvolvimento de interface de um-MED consiste em um trabalho que requer o domínio de conhecimentos de diversas áreas, deve ser, preferencialmente, realizado por uma equipe multidisciplinar. Neste caso, a descrição de uma metodologia torna-se fundamental. Adotando um processo organizado de trabalho, é possível controlar os fatores envolvidos, estabelecer responsabilidades e prazos, melhorar a comunicação entre os membros da equipe e economizar recursos. Conseqüentemente, aumenta-se a probabilidade de que os objetivos pedagógicos propostos no início do projeto sejam mantidos durante o processo e, também, de que o resultado pretendido, no caso a aprendizagem, seja alcançado ao seu final. 
Para Mediante pesquisa realizada (Passos, 2011) observou-se a carência de uma metodologia para MED, focada nas necessidades educacionais, que também considerasse efetivamente aspectos de Design e Engenharia. Este artigo apresenta, portanto, o processo de desenvolvimento da metodologia Interad com base em estudos em Educação, Design e Engenharia de Software, a saber: a metodologia de Amante e Morgado (2001); o Design Instrucional (Filatro, 2008); o Design Pedagógico (Torrezan, 2009); o Design de Interação (Preece, J. Rogers, Y. Sharp, H., 2005); os Elementos da Experiência do Usuário (Garrett, 200); e a Engenharia de Software (Pressman, 2002).

Para tanto, primeiramente, foi proposta uma metodologia básica para aplicação em contexto real e para realização de estudo de caso. Essa metodologia foi utilizada pela equipe do Núcleo de Tecnologia Digital Aplicada à Educação (NUTED/UFRGS) na produção de MEDs durante o ano de 2010. Com base no referencial teórico e nos resultados do estudo de caso foi desenvolvida a metodologia Interad. Para validação da metodologia foi aplicado um curso onde os participantes a utilizaram em projetos de interface de MED. Para apoio ao curso, foi desenvolvido um objeto de aprendizagem que contém a metodologia enriquecida por exemplos e modelos de utilização.

A justificativa para o desenvolvimento de uma metodologia específica para MED está nas diferenças de aplicação entre as interfaces educacionais e as interfaces comerciais. Filatro (2008) afirma que a produção comercial é linear e baseia-se na transmissão unidirecional, ou com baixa interatividade. Enquanto isso, o aprendizado eletrônico, normalmente, propõe percursos diferenciados conforme as respostas dos alunos e apóia-se na lógica da comunicação bi ou multidirecional, estabelecendo interação entre aluno e conteúdo e/ou proporcionando interação entre as pessoas.

Porém, a principal diferença está no produto final. Os recursos comerciais visam comunicar uma mensagem de maneira interessante e atraente. Já no aprendizado eletrônico, os objetivos estão relacionados ao desenvolvimento de habilidades e à construção do conhecimento. Sendo assim, o objetivo maior é educar e provocar mudança de comportamento, o que implica estratégias diferenciadas da indústria de mídia em geral (Filatro, 2008).

\section{Levantamento de dados}

Este item relata o estudo de caso realizado no NUTED durante o ano de 2010. O NUTED consiste em um grupo interdisciplinar de pesquisa que integra estudantes de graduação e pós-graduação de diversas áreas do conhecimento no desenvolvimento e na utilização de tecnologias digitais aplicadas à educação.

Nessa oportunidade, observou-se a rotina de planejamento e construção dos objetos de aprendizagem, bem como a interação entre os membros do grupo de pesquisa. Para tanto, foi proposta uma metodologia preliminar para ser utilizada pela equipe no desenvolvimento dos objetos e avaliada tanto em termos de processo como de resultados obtidos. Objetivou-se, a partir dos dados levantados, desenvolver um processo que fosse baseado em situações e necessidades efetivas de trabalho.

A proposta inicial de metodologia foi preparada segundo o referencial citado no item 1 e contou com 5 fases. Os tópicos dessas fases ainda não apresentavam explicações detalhadas. As fases e os tópicos eram os seguintes: a) Compreensão - busca de subsídios para o projeto, levantamento de informações relativas ao tema, definição dos objetivos pedagógicos, das necessidades do usuário, do público-alvo, da filosofia da instituição, do conceito visual do material e do contexto de aplicação. b) Preparação - descrição de elementos e funcionalidades necessárias para atingir os objetivos pedagógicos propostos; definição de equipe; seleção de recursos interativos e de mídia apropriada; levantamento de conteúdo; c) Experimentação organização do conteúdo conforme a proposta interativa; design de interação; desenho dos fluxos de tarefa; d) Elaboração - projeto gráfico; definição da hierarquia da informação; composição dos elementos gráficos; agrupamento do conteúdo; e) Apresentação - design visual; definição dos atributos gráficos e apresentação de textos e imagens.

Participaram do estudo de caso três alunos de graduação da área da Educação, um aluno do Design Gráfico e um aluno de Artes Visuais. Ainda participou uma arquiteta, doutoranda em Informática na Educação. Suas idades variavam entre 22 e 33 anos, sendo dois do sexo masculino e 4 do sexo feminino. Quanto ao nível de instrução e a ocupação principal, cinco deles 
eram bolsistas de iniciação científica, enquanto uma era professora universitária. A experiência referente ao desenvolvimento de MEDs que eles declararam ter variava entre um e cinco anos.

As ferramentas escolhidas para coleta de dados foram as seguintes: observação participante e entrevistas. A observação participante é indicada para coleta de dados em pesquisas que consideram o contexto dos eventos; que acontecem em tempo real; e onde importa perceber comportamentos interpessoais. As entrevistas, por sua vez, podem enfocar diretamente o tópico do estudo de caso e fornecer inferências causais percebidas (Yin, 2005). Os dados obtidos são resultados do cruzamento dos dados, destacando-se os resultados que foram encontrados tanto nas observações anotadas pelas autoras quanto nas entrevistas realizadas com a equipe.

Os dados levantados mostram os seguintes resultados: a) a equipe tem interesse em uma metodologia que contemple tanto o projeto gráfico, quanto os objetivos pedagógicos, sem pender para um ou outro; b) os integrantes da equipe tiveram dificuldades de entender algumas etapas do trabalho; c) foram requisitados exemplos de tarefas já realizadas e modelos para auxílio na execução das atividades; d) alguns integrantes da equipe tiveram dificuldade em entender termos específicos; e) os integrantes da equipe de formação pedagógica não se envolveram em todas as etapas e não Ihes ficou claro o trabalho integrado; f) a aprovação do material foi tardia, ocorrendo apenas na etapa final; g) não foi feito corretamente o levantamento de informações a respeito da expectativa do coordenador do projeto quanto ao material.

Mediante essas observações, foram propostas as seguintes alterações na metodologia: a) reforçar a parte pedagógica da metodologia inserindo mais etapas que contemplem essa área; b) detalhar a metodologia e fornecer exemplos tornando-a compreensível para todos os integrantes da equipe; c) disponibilizar formulários que orientem a produção; d) prover vocabulário acessível aos profissionais das diferentes áreas que podem estar envolvidas na produção de MEDs; e) reforçar a idéia de que o trabalho da equipe deve ser integrado; f) incluir uma etapa de avaliação na fase Experimentação; g) especificar detalhadamente a atenção que deve ser dada para a expectativa do coordenador do projeto ou solicitante do material.

\section{Metodologia Interad}

A metodologia Interad foi formulada com base nos estudos de Garrett (2003), Amante e Morgado (2001), no Design Instrucional (Filatro, 2008), na Engenharia de Software (Pressman, 2002) e no Design de Interação (Preece, Rogers e Sharp, 2005). Comparando-se esses estudos foi possível destacar as etapas que poderiam ser adaptadas para o desenvolvimento de metodologia específica para o design de interfaces de MEDs. A seguir, o estudo de caso realizado junto ao NUTED (item 2) contribuiu para a observação das necessidades de aplicação prática de uma metodologia desse tipo. A metodologia Interad foi, então, composta a partir da pesquisa teórica aliada aos resultados do estudo de caso.

A metodologia Interad é apresentada em cinco fases: a primeira fase é chamada de Compreensão e apresenta a pesquisa de informações sobre o material a ser projetado; a segunda fase foi denominada de Preparação, pois reúne e sintetiza as informações levantadas; a seguir, a fase Experimentação dá início ao projeto concreto e lança a base de suas estruturas; nas fases Elaboração e Apresentação é executada a interface em termos de wireframe ${ }^{1}$ e design visual, respectivamente. A Figura 1 apresenta um resumo da metodologia Interad com 17 etapas divididas em 5 fases.

1 os wireframes consistem em esboços preliminares das páginas que mostram o esqueleto do sistema de navegação 
Figura 1: Metodologia Interad

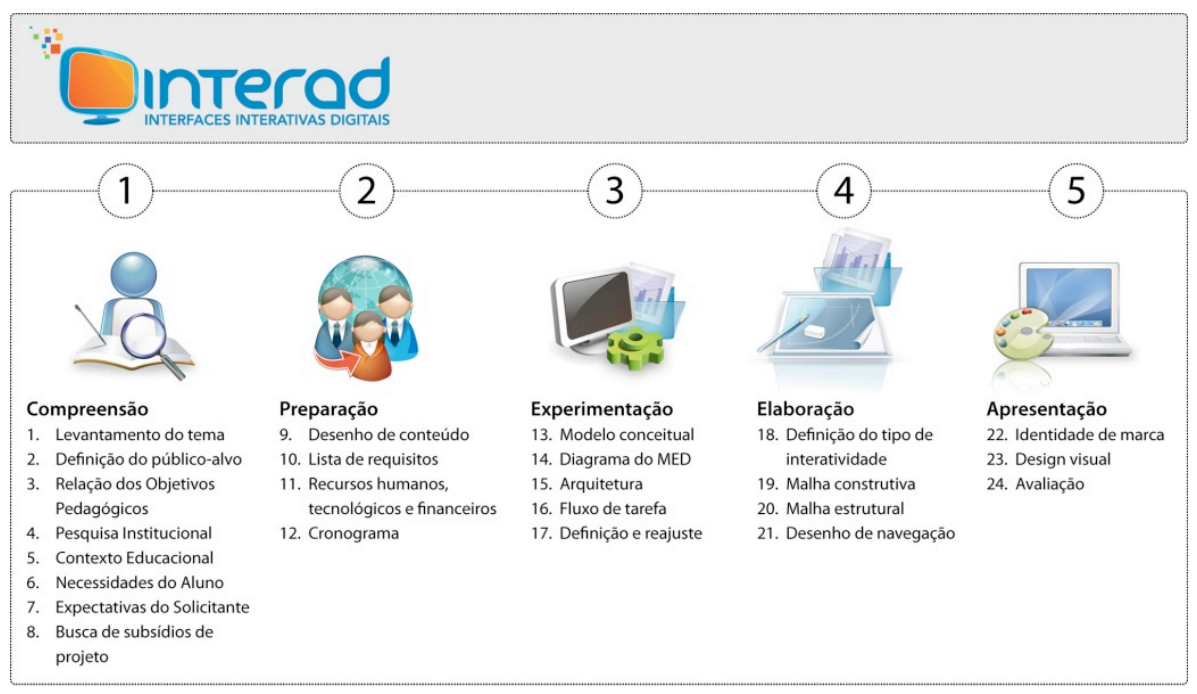

\section{Fase Compreensão}

A primeira fase da metodologia é chamada de Compreensão porque as suas ações se concentram em esclarecer qual o problema de projeto a ser resolvido. Nessa primeira fase estão presentes etapas de levantamento de informações gerais relevantes ao projeto; pesquisa sobre $o$ público-alvo e sobre a instituição que solicitou a produção do MED.

Em termos de experiência do usuário, Garrett (2003) considera que o fundamento do sucesso de um projeto esteja em uma fase inicial claramente articulada. Para isso, é preciso definir as intenções de quem solicitou o projeto e as necessidades do provável usuário. Essas duas questões deverão nortear todas as decisões relativas ao projeto de experiência do usuário. Ele considera que a chave esteja em explicitar com clareza exatamente o que se quer. Quanto mais precisamente são propostas as alternativas viáveis mais provável será que as metas sejam atingidas.

No caso da presente pesquisa, o usuário é o aluno e a organização apresenta objetivos pedagógicos. Dessa forma, é preciso agregar ao projeto de design uma abordagem que considere aspectos de educação. Assim, algumas das etapas iniciais da metodologia Interad são construídas com base nos estudo de Amante e Morgado (2001) e do Design Instrucional (Filatro, 2008). Esses autores evidenciam a importância da definição dos objetivos pedagógicos para o êxito do material; além da pesquisa referente a instituição onde este será produzido; e o contexto educacional onde o será aplicado. Esses dados precisam ser levantados e devidamente relatados antes de iniciar-se o projeto de interface.

Nessa fase ainda são levantadas as necessidades do aluno, enquanto usuário do sistema. São definidas as metas de usabilidade, que pretendem promover um sistema eficiente, eficaz e agradável; e as metas experiência do usuário, que se referem a como ele se sentirá ao utilizar ambiente digital (Preece, Rogers e Sharp, 2005). Por fim, dá-se a busca de subsídios de projeto, que trata de uma pesquisa bibliográfica do projetista em referenciais que poderão enriquecer o seu trabalho (Garrett, 2003).

\section{Fase Preparação}

A fase inicial de um projeto procura resposta para uma pergunta abstrata: "Por que fazer esse material?" Já, a fase seguinte, apresenta uma nova questão: "Como fazer o material?" (Garrett, 2003). A partir dos dados obtidos junto à equipe pedagógica e à instituição solicitante, dos resultados das pesquisas com usuários e das pesquisas bibliográficas, é possível passar para a etapa onde essas informações serão analisadas e sintetizadas. Essa fase trata da transformação das necessidades do aluno em requisitos de projeto através da construção do escopo, ou lista de requisitos. Inicia-se pelo desenho de todo o conteúdo que será disponibilizado no material 
(Pressman, 2002). De posse dessas informações é descrita a lista de requisitos. A fase Preparação se encerra com a definição de recursos e prazos e o estabelecimento do cronograma de atividades.

\section{Fase Experimentação}

A terceira fase da metodologia é chamada Experimentação. Inicialmente, são definidos o modelo conceitual a ser aplicado nos elementos gráficos da interface e a metáfora que poderá ser utilizada. A seguir, é desenhado o diagrama, ou mapa, do MED mediante a escolha do tipo de arquitetura navegacional adequado aos objetivos pedagógicos (Filatro, 2008). Por fim, são desenhados os fluxos de execução das tarefas. Com base no estudo de caso realizado (item 2), a fase Experimentação apresenta ainda uma etapa de definição e reajuste, quando há uma aprovação do diagrama por parte da equipe e da instituição que solicitou o material.

Garrett (2003) afirma que reunir e priorizar requisitos permite obter uma imagem clara dos itens que estarão presentes no produto final, mas que a lista de requisitos, entretanto, não demonstra como as peças trabalham juntas, como um todo coeso. Ele explica que é, então, momento de formar a estrutura conceitual do material, quando as preocupações são transferidas de pesquisas abstratas para fatores concretos que irão determinar a experiência do usuário.

\section{Fase Elaboração}

A fase Elaboração prevê a escolha dos tipos de interatividade que serão mais indicados de acordo com os objetivos pedagógicos propostos e com o perfil dos alunos. Definidos os recursos interativos pretendidos, passa-se às etapas de desenho das malhas construtiva e estrutural. A malha construtiva é um recurso vindo do design editorial que permite uma melhor organização visual da página, enquanto a malha estrutural - ou wireframe - define a distribuição hierárquica dos elementos gráficos. Por fim, o desenho de navegação determina a movimentação do usuário pela página através da escolha e do posicionamento dos links.

\section{Fase Apresentação}

$\mathrm{Na}$ última fase da metodologia tem lugar o design gráfico da identidade de marca do material e dos elementos da interface. Mesmo que se pense na parte visual do projeto como principalmente estética, essa fase envolve também: estratégia de comunicação, legibilidade, usabilidade e funcionalidade. Esses aspectos irão influenciar na identificação do aluno com o assunto tratado, bem como na sua interação com o material e apropriação dos conteúdos. Encerrando o projeto, apresenta-se a avaliação da interface por parte da instituição solicitante para aprovação ou para reajustes.

\section{Objeto de aprendizagem}

O objeto de aprendizagem Interad ${ }^{2}$ foi desenvolvido no NUTED atendendo ao edital 11 da Secretaria de Educação a Distância (SEAD/UFRGS). O objetivo desse recurso é auxiliar na aplicação da metodologia para design de interfaces conduzindo a um projeto pedagogicamente orientado e, ao mesmo tempo atento à parte gráfica e visual.

Cada módulo do Interad apresenta uma fase da metodologia e orienta para a execução de uma parte do projeto de interface de um MED. Os módulos contam com exemplos que auxiliam a detalhar a proposta de projeto, além de material complementar para consulta. O objeto apresenta também roteiros que ajudam o aluno a construir sua própria interface de MED.

Esse material foi projetado para servir como apoio para cursos que apresentassem a metodologia Interad. No entanto, seguindo a lógica de reutilização dos objetos de aprendizagem, é possível que seja utilizado também por equipes de trabalho para orientarem seus projetos; ou por educadores para transmitir a metodologia para suas equipes. Sendo modular, possibilita o

2 http://nuted.edu.ufrgs.br/interad/ 
trabalho nos conteúdos separadamente conforme interesses específicos de projeto. Cabe ainda ressaltar que o objeto Interad foi desenvolvido segundo a metodologia proposta neste trabalho.

\section{Aplicação da metodologia}

Como etapa de validação da pesquisa foi realizado um curso sobre design de interface de MED. Esse curso foi organizado pelo NUTED e ofertado pela Secretaria de Educação a Distância (SEAD/UFRGS). O conteúdo e as atividades do curso consistiram na apresentação e aplicação prática da metodologia Interad. O objeto de aprendizagem Interad foi usado como apoio didático. O perfil dos participantes do curso foi de professores de graduação da UFRGS em áreas diversas. A Figura 2 apresenta a interface da tela inicial do objeto Interad.

Figure 2: Tela inicial do objeto de aprendizagem Interad

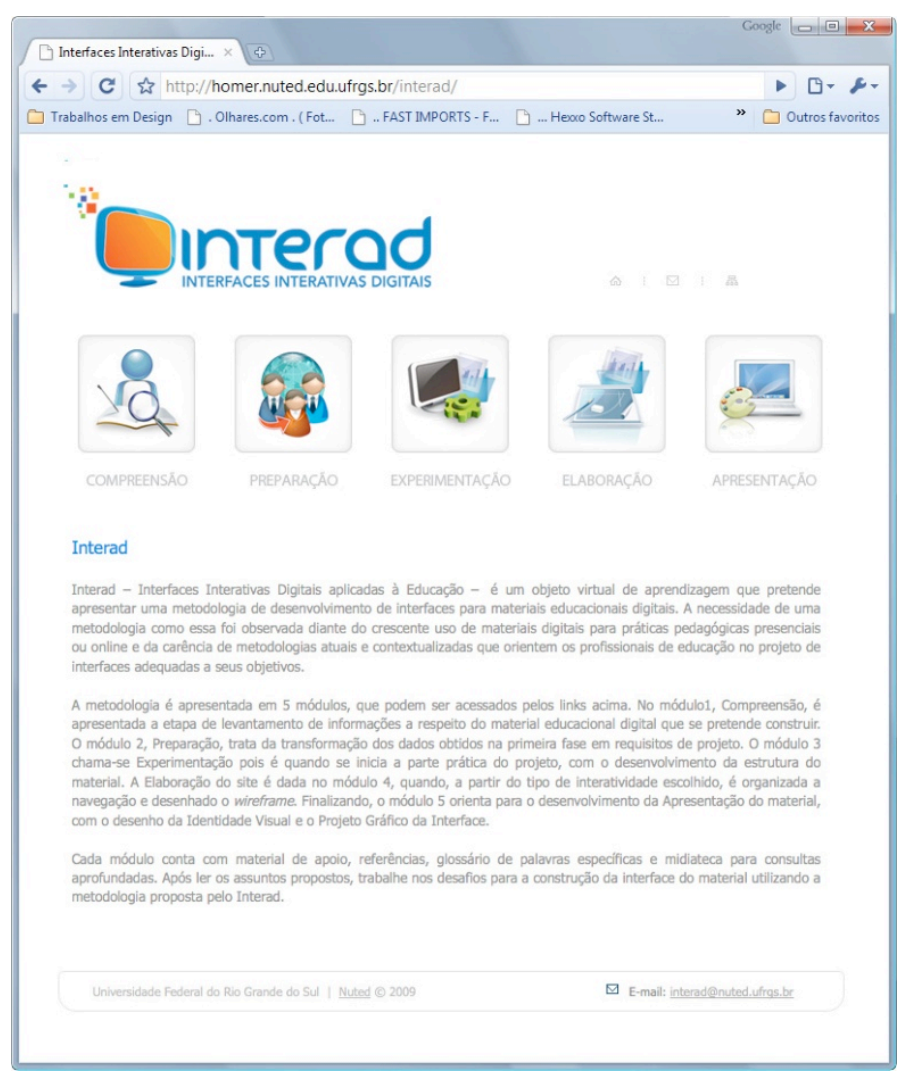

O objetivo pedagógico proposto para o curso foi levar o aluno a refletir sobre a necessidade do trabalho em grupo no desenvolvimento de MEDs (agregando profissionais de diferentes áreas) e do uso de técnicas que favoreçam essa prática. A intenção foi que esse alunos conhecessem o processo e o testassem para produção individual ou em equipe de uma interface de MED.

O curso foi realizado no Laboratório de Informática do Centro Interdisciplinar de Novas Tecnologias na Educação (CINTED/UFRGS) ${ }^{3}$, nos dias 02, 09, 13 e 16 de dezembro de 2010, totalizando 16 horas de aula. Foi organizado pelo NUTED e ofertado pela SEAD/UFRGS.

\footnotetext{
${ }^{3}$ Anexo I da Reitoria - Prédio 12105 - Campus Centro - Av. Paulo Gama, s/n. http://www.cinted.ufrgs.br/
} 
O Moodle ${ }^{4}$ foi utilizado como plataforma de apoio para interação entre os alunos e publicação dos trabalhos.

A partir do curso foram coletados dados referentes ao desempenho dos alunos no processo de aplicação da metodologia; a interação nas equipes de trabalho; ao conteúdo apresentado; e a impressão dos alunos sobre o curso. Foi avaliado também o funcionamento do material. As questões norteadoras da pesquisa foram as seguintes: a) Seguindo a Metodologia Interad os alunos conseguiram participar do processo de design de uma interface de MED? b) O conteúdo disponível no objeto foi adequado e suficiente para a execução dos desafios? c) Os desafios conduziram a uma melhor apreensão do conteúdo? d) Após participarem do curso, os alunos se consideraram mais preparados para trabalhar em equipes de projeto de MEDs? e) Os alunos consideram-se aptos para projetarem interfaces para MEDs com melhor qualidade do que faziam antes do curso?

A coleta de dados foi feita a partir de três ferramentas propostas em Yin (2005), que são as seguintes: observação, entrevistas e documentação. A observação foi realizada através de anotações dos ministrantes do curso sobre o desempenho e os comentários dos alunos em aula. Também foi realizada a gravação da apresentação final dos alunos e avaliação dos colegas. Nas entrevistas, os alunos foram convidados a responder um questionário sobre a metodologia, o objeto e o curso. Alguns deles foram entrevistados individualmente e seus depoimentos foram gravados. As entrevistas foram preparadas conforme orientações de Robson (1993, apud Preece, Rogers e Sharp, 2005). A documentação reunida consistiu nos relatórios de atividades publicados no Webfólio e nas participações em Fóruns realizados durante o curso.

Estiveram presentes no curso cinco alunos. Dentre eles, três alunos tiveram disponibilidade para participar da pesquisa. Para resguardar suas identidades, os entrevistados serão aqui chamadas de A1, A2 e A3. Todos os três são docentes, com idades entre 30 e 50 anos: A1 na área de Ciências Contábeis; A2 em Licenciatura em Computação e A3 em Biologia. Quanto a experiência com MEDs, A1 declara ter interesse mas nunca ter produzido; A2 orienta alunos de Computação para projeto desses recursos; $\mathrm{A} 3$, com auxílio de um bolsista, desenvolve MEDs para apoio a suas aulas presenciais.

As informações levantadas com cada uma das ferramentas (observação, entrevistas e documentação) foram cruzadas para assegurar-se a obtenção de resultados mais precisos conforme orientações de Yin (2005). Os resultados foram organizados mediante a metodologia de análise de conteúdo proposta por Moraes (1999). sendo divididos em 4 categorias: aluno, metodologia, objeto de aprendizagem e curso. Os próximos subitens apresentam os resultados de cada categoria.

\section{Categoria Aluno}

Conforme os dados levantados, os alunos pesquisados, de forma geral, chegaram ao curso com o objetivo de aprender a produzir materiais educacionais digitais ou de melhorar a qualidade de seus projetos. Como aplicação pretendida para o conhecimento adquirido, eles declararam o seguinte: A2, doutorando, declarou que pretende usar a metodologia na produção do material de aprendizagem objeto de sua tese, além de levá-la para seus alunos de graduação, para que os auxilie em seus projetos e para que tenham um guia para escrever artigos científicos sobre os MEDs que produzem; enquanto isso, A1 e A3 pretendem usar a metodologia em seus próximos trabalhos, ressaltando que $A 3$ demonstrou especial interesse em que a metodologia melhore seu trabalho em grupo, facilitando a comunicação entre conteudista e webdesigner. Em relação à aprendizagem, os alunos, sozinhos ou em grupos, preencheram relatórios cumprindo devidamente as tarefas propostas, o que demonstra que conseguiram acompanhar e compreender as etapas da metodologia e que alcançaram os objetivos pedagógicos do curso.

\section{Categoria Metodologia}

A metodologia pretende orientar para o trabalho em equipe, envolvendo diferentes áreas, sendo principalmente: gráficas, tecnológicas e pedagógicas. Tratando de assuntos referentes a essas três áreas básicas, a teoria pode apresentar-se estranha ou complexa em algumas partes

\footnotetext{
${ }^{4}$ https://moodleinstitucional.ufrgs.br/login/index.php
} 
especificas, para determinados participantes de uma equipe. No entanto, os alunos do curso declararam que conseguiram compreender a maioria dos temas apresentados.

Alguns alunos começaram o trabalho em grupos, mas tendo interesse na orientação prestada no curso, preferiram desenvolver trabalhos individuais, com objetivos próprios e aplicáveis em seus trabalhos. Entende-se que essa atitude reafirma seu interesse em adotar a metodologia Interad em seus próximos projetos.

Os alunos declararam, a partir do conhecimento da metodologia Interad, sentirem-se melhor preparados para a construção de MEDs. Acreditam, ainda, que trabalharão com mais eficiência em seus próximos projetos e deverão alcançar resultados de melhor qualidade.

\section{Categoria Objeto de Aprendizagem}

Os alunos manifestaram interesse por um curso mais longo, onde pudessem se aprofundar no assunto e tivessem mais tempo para trabalhar nos desafios que são, cada um, uma etapa do projeto. Isso porque eles estavam aproveitando o curso para desenvolver projetos próprios. Ainda quanto aos desafios, os alunos demonstraram perceber os encadeamentos entre os conteúdos, executando os últimos desafios com base nos primeiros, por exemplo: propondo uma interface (desafio do módulo 5) composta de elementos visuais condizentes com a faixa etária a que se destina o material (definida no desafio do módulo 1).

Quanto à apresentação visual do objeto, bem como aos exemplos de projetos apresentados e aos modelos para execução dos desafios, os alunos afirmaram estarem satisfeitos. Sobre a utilidade do objeto, A1, A2 e A3 declararam que pretendem reutilizá-lo em seus próximos projetos de MED, e A2 ainda deseja apresentá-lo a seus alunos de graduação em Computação.

\section{Categoria Curso}

No primeiro dia do curso de interfaces estavam presentes cinco alunos, que compareceram nas demais aulas e cumpriram o curso até o final. A pesquisa foi feita com os três alunos que se dispuseram para participar das entrevistas. Como dito no item anterior, alguns alunos desejariam que o curso fosse mais longo devido ao seu interesse em produzir MEDs para aplicação prática em suas atividades. $O$ relacionamento entre alunos e ministrantes foi satisfatório e os alunos não apresentaram reclamações. O material utilizado no curso foi bem aceito pelos alunos.

\section{Considerações finais}

A metodologia e o objeto de aprendizagem Interad contribuem para orientar o projeto de interface de MED auxiliando equipes a organizarem o seu trabalho. Conforme atestado pelos participantes da pesquisa, a metodologia é útil para auxiliar no desenvolvimento de interfaces para MEDs e o objeto de aprendizagem apresenta recursos que facilitam o trabalho. A metodologia Interad é restrita à interface do MED, como sugestão para próximas pesquisas, propõe-se que seja desenvolvida uma metodologia para desenvolvimento de todo o MED, incluindo produção de conteúdo e programação.

\section{Referências}

AMANTE, L.; MORGADO, L., 2001. Metodologia de Concepção e Desenvolvimento de Aplicações Educativas: o caso dos materiais hipermedia. In: Discursos, II Série, n especial, PP. 125-138, Universidade Aberta.

BEHAR, P.A. (orgs.), 2009. Modelos Pedagógicos em Educação a Distância. Porto Alegre: Artmed.

FILATRO, A., 2008. Design Instrucional na prática. São Paulo : Pearson Education do Brasil.

GARRETT, J.J., 2003. The elements of user experience: user centered design for the web. New York/Berkeley: Aiga/Nex Riders. 
HARIS, J. Giving You Fitts. Ago. 2006. Acesso em maio de 2010. Disponível em <http://blogs.msdn.com/jensenh/archive/2006/08/22/711808.aspx>.

KALBACH, J., 2009. Design de navegação Web. Porto Alegre: Bookman.

MORAES, Roque, 1999. Análise de conteúdo. Revista Educação, Porto Alegre, v. 22, n.37, p.7-32.

PASSOS, P.C.S.J., 2011. Interad: uma metodologia para design de interfaces de materiais educacionais digitais. Dissertação apresentada ao programa de Pós-Graduação em Educação da Faculdade de Educação da UFRGS.

PIAGET, J., 1974. Aprendizagem e conhecimento. Rio de Janeiro: Freitas Bastos.

PREECE, J. ROGERS, Y. SHARP, H., 2005. Design de Interação: além da interação homemcomputador. Porto Alegre: Bookman.

PRESSMAN, R. S., 2002. Ingeniería del Software: Un enfoque prático. Madrid: Concepción Fernández Madrid.

ROBSON, C.,1993. Real World Research. Oxford, UK: Blackwell.

TORREZZAN, C. A. W., 2009. Design pedagógico: um olhar na construção de materiais educacionais digitais. Dissertação apresentada ao programa de Pós-Graduação em Educação da Faculdade de Educação da UFRGS.

YIN, R., 2005. Estudo de caso: planejamento e métodos. Porto Alegre: Bookman.

\section{Sobre as autoras}

Paula Caroline Schifino Jardim Passos, doutoranda, Programa de Pós-Graduação em Comunicação e Informação da Universidade Federal do Rio Grande do Sul (UFRGS), mestre em Educação pela UFRGS (2011). Especialista em Informática na Educação pela UFRGS (2008). Pesquisa sobre design de interfaces, arquitetura de informação, planejamento gráfico e recursos educacionais digitais.

<paulacarolinejardim@gmail.com>

Patricia Alejandra Behar, doutora, Universidade Federal do Rio Grande do Sul, professora da Faculdade de Educação e dos Cursos de Pós Graduação em Educação (PPGEdu) e em Informática na Educação (PPGIE). Mestre (1991-1993) e Doutora (1994-1998) em Ciência da Computação pela UFRGS. Autora do Livro Modelos Pedagógicos em Educação a Distância. ArtMed: Porto Alegre, 2009.

<pbehar@terra.com.br >

[Artigo recebido em dezembro de 2012, aprovado em dezembro de 2012] 